\title{
Explicit Formulas for Some Generalized Polynomials
}

\author{
Dmitry V. Kruchinin ${ }^{1, *}$ and Vladimir V. Kruchinin ${ }^{2}$ \\ ${ }^{1}$ Department of Complex Information Security, Tomsk State University of Control Systems and Radioelectronics, Tomsk, Russia \\ ${ }^{2}$ Department of Mathematics, Tomsk State University of Control Systems and Radioelectronics, Tomsk, Russia
}

Received: 12 Feb. 2013, Revised: 14 Jun. 2013, Accepted: 17 Jun. 2013

Published online: 1 Sep. 2013

\begin{abstract}
Using notions of composita and composition of generating functions, we establish some explicit formulas for the Generalized Hermite polynomials, the Generalized Humbert polynomials, the Lerch polynomials, and the Mahler polynomials.
\end{abstract}

Keywords: Composita, generating function, Generalized Hermite polynomials, Generalized Humbert polynomials, Lerch polynomials, Mahler polynomials.

\section{Introduction}

The use of polynomials in other areas of mathematics is very impressive: they are important to continued fractions, operator theory, analytic functions, interpolation, approximation theory, numerical analysis, electrostatics, statistical quantum mechanics, special functions, number theory, combinatorics, stochastic processes, sorting and data compression, and etc.

Interesting results in the field of obtaining explicit formulas for polynomials can be found in some recent works by Srivastava [1, 2], Cenkci [3], Boyadzhiev [4] and Kruchinin [5].

In this paper we use the method of obtaining expressions for polynomials based on the composition of generating functions, which was presented by the authors at the 10th International Conference of Numerical Analysis and Applied Mathematics [6].

The generating functions have important roles in many branches of mathematics and mathematical physics. Numerous investigations related to the generating functions for many polynomials can be found in many books and articles (see, for example, [7-15]

The main purpose of this paper is to obtain explicit formulas for the Generalised Hermite polynomials, the Generalised Humbert polynomials, the Lerch polynomials, and the Mahler polynomials.

\section{Preliminary}

In this section, we introduce some basic definitions, operations and notation we need.

In the paper [16] authors introduced the notion of the composita of a given ordinary generating function $F(t)=$ $\sum_{n>0} g(n) t^{n}$.

Suppose $F(t)=\sum_{n>0} f(n) t^{n}$ is the generating function, in which there is no free term $f(0)=0$. From this generating function we can write the following condition

$$
[F(t)]^{k}=\sum_{n>0} F(n, k) t^{n}
$$

The expression $F(n, k)$ is the composita and it is denoted by $F^{\Delta}(n, k)$.

For more information one can see some related works $[5,17,18]$.

Next we show some operations, and rules with compositae.

1.Suppose $F(t)=\sum_{n>0} f(n) t^{n}, B(t)=\sum_{n \geqslant 0} b(n) t^{n}$ are generating functions, and $F^{\Delta}(n, k)$ is the composita of $F(t)$. Then for the composition of generating functions $A(t)=B(F(t))$ the following condition holds

$$
a(n)=\sum_{k=1}^{n} F^{\Delta}(n, k) b(k), \quad a(0)=b(0)
$$

where $A(t)=\sum_{n \geq 0} a(n) t^{n}$.

\footnotetext{
*Corresponding author e-mail: kruchininDm@gmail.com
} 
2.Suppose $F(t)=\sum_{n>0} f(n) t^{n}, G(t)=\sum_{n>0} g(n) t^{n}$ are generating functions, and $F^{\Delta}(n, k), G^{\Delta}(n, k)$ are their compositae, respectively. Then for the generating function $A(t)=F(t)+G(t)$ the composita is equal to

$$
\begin{aligned}
& A^{\Delta}(n, k)=F^{\Delta}(n, k)+G^{\Delta}(n, k)+ \\
& \quad+\sum_{j=1}^{k-1}\left(\begin{array}{l}
k \\
j
\end{array}\right) \sum_{i=j}^{n-k+j} F^{\Delta}(i, j) G^{\Delta}(n-i, k-j) .
\end{aligned}
$$

3.Suppose $F(t)=\sum_{n>0} f(n) t^{n}, G(t)=\sum_{n>0} g(n) t^{n}$ are generating functions, and $F^{\Delta}(n, k), G^{\Delta}(n, k)$ are their compositae, respectively. Then for the composition of generating functions $A(t)=G(F(t))$ the composita is equal to

$$
A^{\Delta}(n, k)=\sum_{m=k}^{n} F^{\Delta}(n, m) G^{\Delta}(m, k)
$$

\section{Generalized Hermite polynomials}

In this section we consider the generalization of the Hermite polynomials and obtain some interesting identities for these polynomials.

The Generalised Hermite polynomials are polynomials that arise in many different fields, for instance in quantum mechanics, optical systems, kinetic theory of gases, theories of fluctuations [19-21].

There exist vast investigations concerned with Hermite polynomials, for example, Dattoli [22], Subuhi Khan et al. [23, 24] study summation formulae; Brafman [25], Lahiri [26], Gould and Hopper [27], Dattoli [28] study the generalization of Hermite polynomials.

Using the notion of the composita and the generating functions for the Generalized Hermite polynomials, we get explicit representations.

The Gould-Hopper generalized Hermite polynomials are defined by the following generating function

$$
\sum_{n \geq 0} g_{n}^{m}(x, h) \frac{t^{n}}{n !}=\exp \left(x t+h t^{m}\right)
$$

where $m$ is a positive integer.

First, we obtain the composita of the generating function

$$
F(x, m, h, t)=\left(x t+h t^{m}\right)
$$

as the coefficients with respect to $t^{n}$ in $F^{k}(x, m, h, t)$, where $m \geq 1$ is integer and the other parameters are unrestricted.

Applying the binomial theorem, we have

$F^{k}(x, m, h, t)=t^{k}\left(x+h t^{m-1}\right)^{k}=t^{k} \sum_{j=0}^{k}\left(\begin{array}{l}k \\ j\end{array}\right) x^{k-j} h^{j} t^{j(m-1)}$.
Substituting $n=j(m-1)+k$, we obtain the composita of $F(x, m, h, t)$

$$
F^{\Delta}(n, k, x, m, h)= \begin{cases}\left(\begin{array}{c}
k \\
\frac{n-k}{m-1}
\end{array}\right) x^{k-\frac{n-k}{m-1}} h^{\frac{n-k}{m-1}}, & \text { if } \frac{n-k}{m-1} \in \mathbb{N}, \\
0, & \text { otherwise, }\end{cases}
$$

where $n \geq k$.

Below we present few first terms of the composita in a triangular form for the case $m=3$

$$
\begin{aligned}
& h^{0} \quad 0^{x} x^{3} \\
& \begin{array}{llll}
0 & 2 h x & 0 & x^{4}
\end{array} \\
& \begin{array}{lllll}
0 & 0 & 3 h x^{2} & 0 & x^{5}
\end{array}
\end{aligned}
$$

For case $m=1$, the composita is

$$
F^{\Delta}(n, k, x, 1, h)=\left(\begin{array}{c}
k \\
j
\end{array}\right) \delta_{n, k} x^{k-j} h^{j}
$$

where $\delta_{n, k}$ is the Kronecker delta,

$$
\delta_{n, k}= \begin{cases}1, & \text { if } n=k \\ 0, & \text { if } n \neq k\end{cases}
$$

Therefore, according to (2), the expression for the Gould-Hopper generalized Hermite polynomials is

$$
g_{n}^{m}(x, h)=n ! \sum_{k=1}^{n} \frac{1}{k !} F^{\Delta}(n, k, x, m, h)
$$

or making few operations, we get the Gould-Hopper explicit representation (cf. [27])

$$
g_{n}^{m}(x, h)=n ! \sum_{r=0}^{\left[\frac{n}{m}\right]} \frac{x^{n-m r} h^{r}}{r !(n-m r) !} .
$$

Gould and Hopper also indicated another generalization of the Hermite polynomials by the generating function

$$
\sum_{n \geq 0} H_{n}^{r}(x, a, p) \frac{t^{n}}{n !}=\left(1-\frac{t}{x}\right)^{a} \exp \left(p\left(x^{r}-(x-t)^{r}\right)\right) .
$$

Let us consider the generating function as a product of two generating functions

$$
\left(1-\frac{t}{x}\right)^{a}
$$

and

$$
\exp \left(p\left(x^{r}-(x-t)^{r}\right)\right) .
$$

First we obtain the composita of $p\left(x^{r}-(x-t)^{r}\right)$. The coefficients of $(x-t)^{r}$ are equal to

$$
(-1)^{n}\left(\begin{array}{l}
r \\
n
\end{array}\right) x^{r-n}
$$


Then the composita of the required function is

$$
p^{k} \sum_{j=0}^{k}\left(\begin{array}{l}
k \\
j
\end{array}\right)(-1)^{n+j}\left(\begin{array}{c}
j r \\
n
\end{array}\right) x^{k r-n}
$$

Using (2), we get the expression for coefficients of the generating function

$$
\begin{gathered}
\exp \left(p\left(x^{r}-(x-t)^{r}\right)\right) \\
\sum_{k=0}^{n} \frac{p^{k}\left(\sum_{j=0}^{k}\left(\begin{array}{c}
k \\
j
\end{array}\right)(-1)^{n+j}\left(\begin{array}{c}
j r \\
n
\end{array}\right)\right) x^{k r-n}}{k !} .
\end{gathered}
$$

Coefficients of the generating function

$$
\left(1-\frac{t}{x}\right)^{a}
$$

are specified by the following expression

$$
\left(\begin{array}{l}
a \\
n
\end{array}\right)(-1)^{n} x^{-n}
$$

Therefore, multiplying both expressions, we obtain the following formula for the generalized Hermite polynomials

$$
H_{n}^{r}(x, a, p)=n ! \sum_{i=0}^{n}\left(\begin{array}{c}
a \\
n-i
\end{array}\right) \sum_{k=0}^{i} p^{k} \sum_{j=0}^{k} \frac{(-1)^{n+j}\left(\begin{array}{c}
j r \\
i
\end{array}\right)}{(k-j) ! j !} x^{k r-n}
$$

or according to Gould and Hopper [27], the explicit formula for this case is

$H_{n}^{r}(x, a, p)=(-1)^{n} n ! \sum_{k=0}^{n} p^{k} \frac{x^{r k-n}}{k !} \sum_{j=0}^{k}(-1)^{j}\left(\begin{array}{c}k \\ j\end{array}\right)\left(\begin{array}{c}a+r j \\ n\end{array}\right)$

Now we consider the generalization to the multivariable case, which is introduced by Dattoli et. al in [28] and using the notion of the composita, we obtain the explicit representation. The multivariable generalized Hermite polynomials are defined by the following generating function

$$
\exp \left(2 x t-t^{2}+2 y t^{m}-t^{2 m}\right)=\sum_{n \geq 0}^{(m)} H_{n}(x, y) \frac{t^{n}}{n !}
$$

or by the ordinary Hermite polynomials

$$
{ }^{(m)} H_{n}(x, y)=n ! \sum_{n=0}^{[n / m]} \frac{H_{n-m r}(x) H_{r}(y)}{(n-m r) ! r !} .
$$

We start with calculation the composita of

$$
G(y, m, t)=2 y t^{m}-t^{2 m}
$$

Applying the binomial theorem, we have

$G^{k}(y, m, t)=t^{m k}\left(2 y-t^{m}\right)^{k}=t^{m k} \sum_{j=0}^{k}\left(\begin{array}{c}k \\ j\end{array}\right)(2 y)^{k-j}(-1)^{j} t^{j m}$.
Substituting $n=(k+j) m$, we obtain the composita of $G(y, m, t)$

$$
G^{\Delta}(n, k, y, m)= \begin{cases}\left(\begin{array}{c}
k \\
\frac{n-k m}{m}
\end{array}\right)(-1)^{\frac{n-k m}{m}}(2 y)^{2 k-\frac{n}{m},}, & \text { if } \frac{n-m k}{m} \in \mathbb{N} \\
0, & \text { otherwise. }\end{cases}
$$

According to (6), the composita of $F(x, t)=2 x t-t^{2}$ is

$$
F^{\Delta}(n, k, 2 x, 2,-1)=\left(\begin{array}{c}
k \\
n-k
\end{array}\right)(2 x)^{2 k-n}(-1)^{n-k} .
$$

Using (3) and (2), we obtain the expression for the multivariable generalized Hermite polynomials

$$
\begin{aligned}
& { }^{(m)} H_{n}(x, y) t^{n}=\sum_{k=1}^{n} \frac{n !}{k !}\left(F^{\Delta}(n, k, 2 x, 2,-1) G^{\Delta}(n, k, y, m)+\right. \\
+ & \left.\sum_{j=1}^{k-1}\left(\begin{array}{l}
k \\
j
\end{array}\right) \sum_{i=j}^{n-k+j} F^{\Delta}(i, j, 2 x, 2,-1) G^{\Delta}(n-i, k-j, y, m)\right)
\end{aligned}
$$

where $n>0$.

\section{Generalized Humbert polynomials}

In this section we apply the notion of composita to get explicit formulas for the generalized Humbert polynomials. In 1965, Gould [29] defined the generalized Humbert polynomial $P_{n}(m, x, y, p, C)$ by means of generating function

$$
\left(C-m x t+y t^{m}\right)^{p}=\sum_{n \geq 0} P_{n}(m, x, y, p, C) t^{n},
$$

where $m \geq 1$ is integer and the other parameters are unrestricted.

Changing the parameters in (16) by appropriate way, one can obtain the generating functions for the following polynomials: the Gegenbauer polynomials, the Legendre polynomials, the Humbert polynomials, and many others. form

Represent the generating function in the following

$$
\left(C-m x t+y t^{m}\right)^{p}=C^{p}\left(1-\frac{1}{C}\left(m x t-y t^{m}\right)\right)^{p} .
$$

According to (6), the composita of $\frac{1}{C}\left(m x t-y t^{m}\right)$ is

$$
F^{\Delta}(n, k, x, m, h, C)=\frac{1}{C^{k}}\left(\begin{array}{c}
k \\
\frac{n-k}{m-1}
\end{array}\right)(m x)^{k-\frac{n-k}{m-1}}(-y)^{\frac{n-k}{m-1}}
$$

where $\frac{n-k}{m-1} \in \mathbb{N}$ and $n \geq k$.

Coefficients of the generating function

$$
(1-x)^{p}
$$


are specified by the following expression

$$
\left(\begin{array}{l}
p \\
n
\end{array}\right)(-1)^{n}
$$

Therefore, according to (2) and substituting $j=\frac{n-k}{m-1}$, we obtain

$$
\begin{array}{r}
P_{n}(m, x, y, p, C)=\sum_{j=0}^{\left[\frac{n}{m}\right]}\left(\begin{array}{c}
p \\
n-(m-1)
\end{array}\right) \frac{(-m x)^{n-m j} y^{j}}{C^{n-(m-1) j-p}} \times \\
\times\left(\begin{array}{c}
n-(m-1) j \\
j
\end{array}\right)
\end{array}
$$

or making few operations, we get the Gould explicit representation (cf. [29])

$$
\begin{aligned}
P_{n}(m, x, y, p, C)= & \sum_{k=0}^{\left[\frac{n}{m}\right]}\left(\begin{array}{c}
p \\
k
\end{array}\right)\left(\begin{array}{c}
p-k \\
n-m k
\end{array}\right) \times \\
& \times C^{p-n+(m-1) k} y^{k}(-m x)^{n-m k} .
\end{aligned}
$$

Next we obtain the explicit formula another way. We represent the generating function (16) as the following composition of generating functions

$$
\left(1-m x t+y t^{m}\right)^{p}=C^{p} \exp \left(p \ln \left(1+\frac{1}{C} h(x, m, y, t)\right)\right),
$$

where $h(x, m, t)=-m x t+y t^{m}$.

From (6), the composita of $\frac{1}{C} h(x, m, y, t)$ is equal to

$$
\begin{cases}\frac{1}{C^{k}}\left(\begin{array}{c}
k \\
\frac{n-k}{m-1}
\end{array}\right)(-m x)^{k-\frac{n-k}{m-1}} y^{\frac{n-k}{m-1}}, & \text { if } \frac{n-k}{m-1} \in \mathbb{N}, \\
0, & \text { otherwise. }\end{cases}
$$

According to Comtet [18], the following expression holds true:

$$
\sum_{n \geq k} s(n, k) \frac{t^{n}}{n !}=\frac{1}{k !} \ln ^{k}(1+t),
$$

where $s(n, k)$ are the Stirling numbers of the first kind.

Then the composita of $\ln (1+t)$ is

$$
\frac{k !}{n !} s(n, k)
$$

Using (4) and substituting $i=\frac{n-j}{m-1}$, we obtain the composita of $p \ln \left(1+\frac{1}{C} h(x, m,, y t)\right)$

$$
\begin{aligned}
(p)^{k} \sum_{i=0}^{\left[\frac{n-k}{m-1}\right]} \frac{y^{i}(-m x)^{n-i m} k !}{C^{n-i(m-1)}(n-i(m-1)) !} & \left(\begin{array}{c}
n-i(m-1) \\
i
\end{array}\right) \times \\
& \times s(n-i(m-1), k) .
\end{aligned}
$$

Therefore, according (2), the coefficients with respect to $t$ for the generating function
$C^{p} \exp \left(p \ln \left(1+\frac{1}{C} h(x, m, y, t)\right)\right)$ are determined by the expression

$$
\begin{aligned}
P_{n}(m, x, y, p, C)=\sum_{k=0}^{n}(p)^{k} & \sum_{i=0}^{\left[\frac{n-k}{m-1}\right]} \frac{y^{i}(-m x)^{n-i m}}{C^{n-i(m-1)-p}(n-i(m-1)) !} \times \\
& \times\left(\begin{array}{c}
n-i(m-1) \\
i
\end{array}\right) s(n-i(m-1), k) .
\end{aligned}
$$

\section{Lerch polynomials}

The Lerch polynomials are defined by the following generating function (see, for details, [7,30])

$$
(1-x \ln (1+t))^{-\lambda}=\sum_{n \geqslant 0} \Phi_{n}(x, \lambda) t^{n} .
$$

From (18) the composita of $x \ln (1+t)$ is equal to

$$
\frac{k !}{n !} s(n, k) x^{k}
$$

We know that for the generating function $\left(\frac{1}{1-x}\right)^{\lambda}$ the coefficients are determined by the expression

$$
\left(\begin{array}{c}
n+\lambda-1 \\
n
\end{array}\right)
$$

Therefore, according to (2), we obtain

$$
\Phi_{n}(x, \lambda)=\sum_{k=0}^{n}\left(\begin{array}{c}
k+\lambda-1 \\
k
\end{array}\right) \frac{k !}{n !} s(n, k) x^{k} .
$$

\section{Mahler polynomials}

The Mahler polynomials are defined by the following generating function (see [8])

$$
e^{x\left(1+t-e^{t}\right)}=\sum_{n \geqslant 0} s_{n}(x) \frac{t^{n}}{n !} .
$$

According to Comtet [18], the following expression holds true:

$$
\sum_{n \geq k} S(n, k) \frac{t^{n}}{n !}=\frac{1}{k !}\left(e^{t}-1\right)^{k}
$$

where $S(n, k)$ are the Stirling numbers of the second kind.

Then the composita of $e^{t}-1$ is

$$
\frac{k !}{n !} S(n, k)
$$

According to [16], the composita of $G(t)=t$ is

$$
G^{\Delta}(n, k)=\delta_{n, k},
$$


where $\delta_{n, k}-$ the Kronecker delta.

Using (3), the composita of the sum of the generating functions $G(t)=t$ and $F(t)=-\left(e^{t}-1\right)$ is equal to

$$
\delta_{n, k}+\sum_{j=1}^{k-1}\left(\begin{array}{l}
k \\
j
\end{array}\right) \sum_{i=j}^{n-k+j} \frac{j !}{i !}(-1)^{j} S(i, j) \delta_{n-i, k-j}+\frac{k !}{n !} S(n, k) .
$$

Since

$$
\delta_{n-i, k-j}= \begin{cases}1, & \text { if } n-i=k-j \\ 0, & \text { otherwise }\end{cases}
$$

we get

$$
\sum_{j=0}^{k}\left(\begin{array}{l}
k \\
j
\end{array}\right) \frac{j !(-1)^{j}}{(n-k+j) !} S(n-k+j, j) .
$$

Therefore, using (2), we obtain

$$
s_{n}(x)=\sum_{k=0}^{n} x^{k}\left(\sum_{j=0}^{k}(-1)^{j}\left(\begin{array}{c}
n \\
k-j
\end{array}\right) S(n-k+j, j)\right) .
$$

For instance, the coefficients of the Mahler polynomials are considered as a triangle of coefficients of degrees $x$ in the sequence $A 137375$ [31].

\section{Acknowledgement}

The reported study was partially supported by the Ministry of education and science of Russia, research project No.7.701.2011(PROJECT 1/12) "Research and development of methods and technologies of the information security in technical and high-performance computing systems".

The authors would like to thank the anonymous referees for their helpful comments and suggestions which improved the original version of this manuscript.

\section{References}

[1] H. M. Srivastava and Guo-Dong Liu, Explicit formulas for the Norlund polynomials $B_{n}^{(x)}$ and $b_{n}^{(x)}$, Computers Math. Applic. 51, 1377-1384 (2006).

[2] H. M. Srivastava and P. G. Todorov, An explicit formula for the generalized Bernoulli polynomials, J. Math. Anal. Appl., 130, 509-513 (1988).

[3] M. Cenkci, An explicit formula for generalized potential polynomials and its applications, Discrete Mathematics, 309, 1498-1510 (2009).

[4] K. N. Boyadzhiev, Derivative polynomials for Tanh, Tan, Sech and Sec in explicit form, Fibonacci Quarterly, 45, 291303 (2007).

[5] D. V. Kruchinin and V. V. Kruchinin, Application of a composition of generating functions for obtaining explicit formulas of polynomials, J. Math. Anal. Appl., 404, 161-171 (2013).
[6] D. V. Kruchinin and V. V. Kruchinin, A method for obtaining expressions for polynomials based on a composition of generating functions, Numerical Analysis and Applied Mathematics ICNAAM 2012, AIP Conf. Proc., 1479, 383386 (2012).

[7] R. P. Jr. Boas and R. C. Buck, Polynomial Expansions of Analytic Functions, Springer-Verlag, (1964).

[8] S. Roman, The Umbral Calculus, Academic Press, (1984).

[9] H. M. Srivastava and H. L. Manocha, A Treatise on Generating Functions, Halsted Press (Ellis Horwood Limited, Chichester), John Wiley and Sons, New York, Chichester, Brisbane and Toronto, (1984).

[10] H. M. Srivastava and J. Choi, Zeta and q-Zeta Functions and Associated Series and Integrals, Elsevier Science Publishers, Amsterdam, London and New York, (2012).

[11] H. M. Srivastava, Some generalizations and basic (or $q$-) extensions of the Bernoulli, Euler and Genocchi polynomials, Applied Mathematics and Information Sciences, 5, 390-444 (2011).

[12] Y. Simsek and M. Acikgoz, A new generating function of (q) Bernstein-type polynomials and their interpolation function. Abstr. Appl. Anal., (2010).

[13] H. Ozden, Y. Simsek, and H. M. Srivastava, A unified presentation of the generating functions of the generalized Bernoulli, Euler and Genocchi polynomials. Computers Math. Applic., 60, 2779-2787 (2010).

[14] R. Dere and Y. Simsek, Applications of umbral algebra to some special polynomials, Adv. Studies Contemp. Math., 22, 433-438 (2012).

[15] Y. Simsek, Complete sum of products of (h,q)-extension of Euler polynomials and numbers, Journal of Difference Equations and Applications, 16, 1331-1348 (2010).

[16] V. V. Kruchinin and D. V. Kruchinin, Composita and its properties, Applicable Analysis and Discrete Mathematics under review, (2013).

[17] D. V. Kruchinin and V. V. Kruchinin, A method for obtaining generating functions for central coefficients of triangles, Journal of Integer Sequences, 15, 1-10 (2012).

[18] L. Comtet, Advanced Combinatorics, D. Reidel Publishing Company, (1974).

[19] V. V. Dodonov, Asymptotic formulae for two-variable Hermite polynomials, Phys. A Math. Gen., 27, 6191-6203 (1994).

[20] G. Dattoli, S. Lorenzutta, G. Maino, and A. Torre, Phasespace dynamics and Hermite polynomials of two variables and two indices, J. Math. Phys., 35, 4451-4462 (1994).

[21] G. Dattoli, S. Lorenzutta, G. Maino, A. Torre, and C. Cesarano, Generalized Hermite polynomials and superGaussian forms, J. Math. Anal. Appl., 203, 597-609 (1996).

[22] G. Dattoli, Summation formulae of special functions and multivariable Hermite polynomials, Nuovo Cimento Soc. Ital. Fis. B., 119, 479-488 (2004).

[23] Subuhi Khan, M. A. Pathan, Nader Ali Makboul Hassan, and Ghazala Yasmin, Implicit summation formulae for Hermite and related polynomials, J. Math. Anal. Appl., 344, 408-416 (2008).

[24] Subuhi Khan and Mustafa Walid Al-Saad, Summation formulae for GouldHopper generalized Hermite polynomials, Computers Math. Applic., 61, 1536-1541 (2011).

[25] F. Brafman, Some generating functions for Laguerre and Hermite polynomials, Canad. J . Math., 9, 180-187 (1957). 
[26] M. Lahiri, On a generalisation of Hermite polynomials, Proc. Amer. Math. Soc., 27, 117-121 (1971).

[27] H. W. Gould and A. T. Hopper, Operational formulas connected with two generalizations of Hermite polynomials, Duke Math. J., 29, 51-63 (1962).

[28] G. Dattoli, C. Chiccoli, S. Lorenzutta, G. Maino, and A. Torre, Theory of generalized Hermite polynomials, Computers Math. Applic., 28, 71-83 (1994).

[29] H. W. Gould, Inverse series relations and other expansions involving Humbert polynomials, Duke Math. J., 32, 697-711 (1965).

[30] H. Bateman, Polynomials associated with those of Lerch, Monatshefte fur Mathematik und Physik, 43, 75-80 (1936).

[31] N. J. Sloane. The On-line Encyclopedia of Integer Sequences, published electronically at http://oeis.org/, (2013).

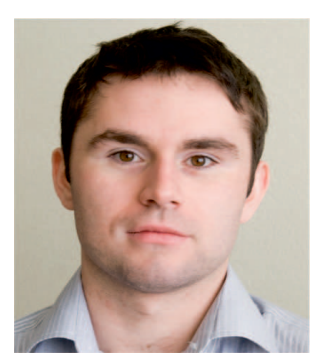

\section{Dmitry V. Kruchinin} is a Ph.D. student at the Tomsk State University of Control Systems and Radioelectronics. His research fields include theory of generating functions, integer sequences, functional equations, polynomials, primality tests, and algorithmic number theory. In June 2011, he graduated with honors from the Tomsk State University of Control Systems and Radioelectronics owith a degree in information security. He was awarded the Presidential Scholarship for Ph.D. students in November, 2012, by the President of the Russian Federation. He has published more than 10 papers.

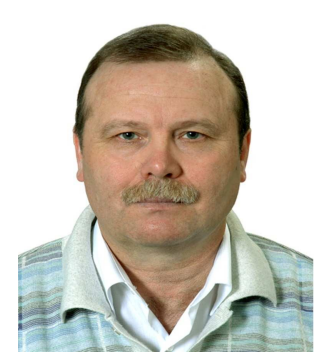

Vladimir V. Kruchinin is a Doctor and Professor of Mathematics at the Tomsk State University of Control Systems and Radioelectronics. He has held the chair of the Department of Mathematics at the Tomsk State University of Control Systems and Radioelectronics since September, 2012. In 2010, he received the degree of a doctor of sciences from the Tomsk State University of Control Systems and Radioelectronics. His main research interests are in the areas of combinatorial generation, theory of partitions, generating functions, integer sequences, functional equations, and polynomials. He is the author of four textbooks and more than 140 publications. 